Hemangioma capilar duodenal como causa atípica de ferropenia. Caso clínico

\title{
Duodenal capillary hemangioma as an atypical cause of iron deficiency. Case report
}

https://doi.org/10.23938/ASSN.0916

\author{
L. Zabalza ${ }^{1}$, V. Jusué ${ }^{1}$, D. Martínez-Acitores ${ }^{1}$, V. Ubieto' ${ }^{1}$, L. Aburruza² ${ }^{\text {, }}$ \\ G. González ${ }^{3}$, G. De Lima ${ }^{4}$, J.J. Vila ${ }^{1}$
}

\section{RESUMEN}

Los hemangiomas son tumores gastrointestinales de naturaleza vascular y benignos. Son infrecuentes, se localizan en la submucosa, y su presentación varía desde casos asintomáticos hasta casos con obstrucción intestinal, anemia o ferropenia. Su diagnóstico inicial suele ser endoscópico a pesar de no presentar características patognomónicas. Tradicionalmente su tratamiento ha sido quirúrgico, si bien en la actualidad es posible su resección endoscópica. Presentamos un caso de ferropenia resuelta tras resección endoscópica de un hemangioma capilar duodenal.

Palabras clave. Hemangioma. Ferropenia. Duodeno.

\begin{abstract}
Hemangiomas are benign vascular gastrointestina tumours. They are infrequent, located in the submucosa, and can be asymptomatic or cause intestinal obstruction, anaemia or ferropenia. Their initial diagnosis is usually endoscopic despite the absence of pathognomonic features. Traditionally their treatment has been surgical although endoscopic resection is now possible. We present a case of ferropenia resolved after endoscopic resection of a capillary duodenal hemangioma.
\end{abstract}

Keywords. Hemangioma. Ferropenia. Duodenum.
1. Servicio de Digestivo. Complejo Hospitalario de Navarra. Pamplona.

2. Servicio de Digestivo. Hospital Universitario Donostia. San Sebastián.

3. Servicio de Digestivo. Hospital Universitario de Burgos. Burgos.

4. Servicio de Anatomía Patológica. Complejo Hospitalario de Navarra. Pamplona.

\section{Correspondencia:}

Lucía Zabalza San Martín

Servicio de Digestivo

Complejo Hospitalario de Navarra

C/ Irunlarrea, 3

31008 Pamplona

E-mail: luciazabalzasanmartin@gmail.com

Recepción: 26/01/2020

Aceptación provisional: 14/03/2020

Aceptación definitiva: 31/07/2020 


\section{INTRODUCCIÓN}

Los hemangiomas son lesiones congénitas consideradas por algunos expertos hamartomas y por otros verdaderas neoplasias. Son lesiones submucosas vasculares benignas que constituyen un hallazgo muy infrecuente en el tracto gastrointestinal y que pueden ser causa de patología tan frecuente como la anemia y/o la ferropenia ${ }^{1}$. Histológicamente existen cuatro tipos, siendo el más frecuente el tipo II o cavernomatoso. Su tratamiento tradicionalmente ha sido quirúrgico aunque en la actualidad generalmente es endoscópico ${ }^{2}$.

Presentamos el caso de una mujer con ferropenia resulta tras polipectomía de una lesión polipoidea duodenal compatible con hemangioma capilar o tipo III.

\section{CASO CLÍNICO}

Mujer de 54 años, valorada en consulta de Digestivo por hallazgo de ferropenia en una analítica realizada por otro motivo. Presentaba ritmo deposicional diario sin productos patológicos y negaba dolor abdominal, pérdida de peso, astenia u otra sintomatología acompañante. La exploración física fue anodina, presentando un abdomen blando, depresible, no doloroso a la palpación con peristaltismo de progresión y sin visceromegalias. Analíticamente, se observó hemoglobina normal con ferropenia, y tirotropina, serología de enfermedad celiaca, vitamina B12 y folato dentro del rango de la normalidad. Se so- licitó gastroscopia y colonoscopia para estudio de la ferropenia.

En la gastroscopia no se observaron alteraciones en las mucosas esofágica y gástrica. Se tomaron biopsias de cuerpo y antro que resultaron normales y Helicobacter pylori negativo. En la segunda porción duodenal, inmediatamente distal a la papila, se objetivó una lesión sésil de $10 \mathrm{~mm}$ que se extirpó en un fragmento con asa de diatermia (Fig. 1A).

Histológicamente se observaron fragmentos de mucosa duodenal de arquitectura polipoidea, reconociéndose una neoformación benigna constituida por vasos ectásicos de pequeño calibre revestidos por endotelio sin atipia citológica (Fig. 1B). Se realizaron técnicas de inmunohistoquímica complementarias observando que las células endoteliales expresaban positividad inmunohistoquímica para los marcadores vasculares Fli-1 y CD31, y negatividad para el marcador vascular linfático D240, compatible todo ello con hemangioma capilar duodenal.

Se completó el estudio con una colonoscopia, observando únicamente hemorroides internas inactivas.

Se realizó control analítico al mes de la polipectomía donde no presentaba ferropenia.

\section{DISCUSIÓN}

Los hemangiomas son lesiones vasculares submucosas muy infrecuentes y constituyen el $0,3 \%$ de los tumores gastrointestinales.

Su localización más frecuente en el tracto gastrointestinal es el intestino delgado
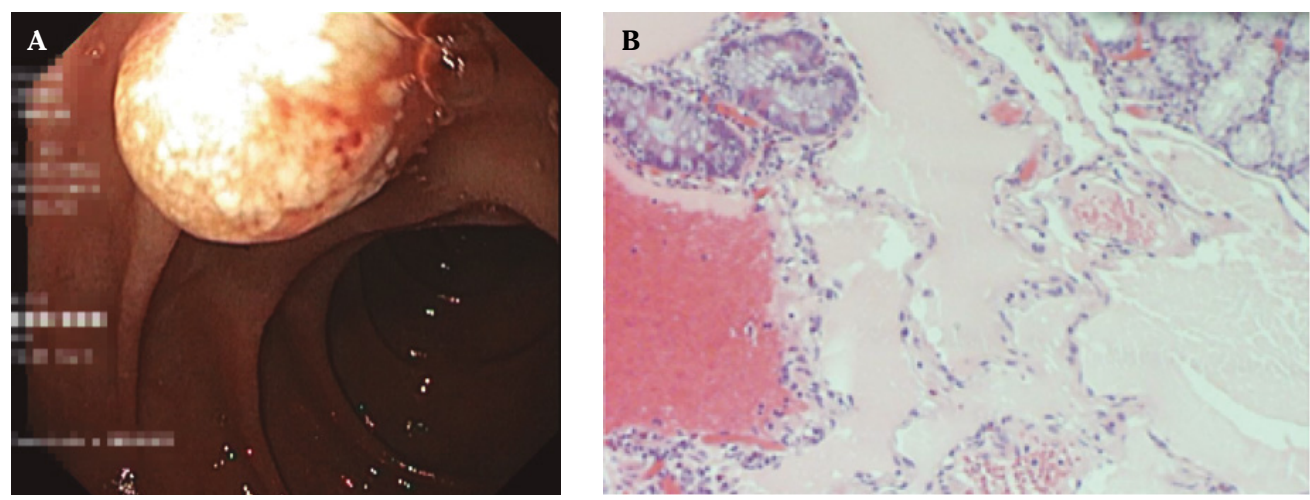

Figura 1. A. Gastroscopia. Lesión polipoidea sésil localizada en duodeno, de $10 \mathrm{~mm}$ de tamaño. B. Imagen histológica. Neoformación benigna constituida por vasos ectásicos de pequeño calibre revestidos por endotelio sin atipia citológica. Hematoxilina-eosina (200x). 
(yeyuno e íleon), seguido del colon y el recto. El hallazgo de estas lesiones a nivel de intestino delgado es cada vez más habitual debido a una mayor utilización de la enteroscopia de doble balón y la cápsula endoscópica ${ }^{3}$. El diagnóstico en el presente caso, al localizarse en duodeno, fue mediante gastroscopia. Pueden manifestarse como hemorragia digestiva alta (melenas o hematemesis), baja (rectorragia), obstrucción intestinal (dolor cólico y vómitos), de manera asintomática, e inusualmente, como en la paciente de este caso, en forma de anemia o ferropenia.

A nivel estructural se caracterizan por poseer un exceso de vasos sanguíneos generalmente venas y capilares y, con frecuencia, son lesiones pequeñas de hasta unos $2 \mathrm{~cm}$ aproximadamente ${ }^{3}$, ambas características presentes en este caso.

El diagnóstico inicial del hemangioma capilar duodenal suele ser endoscópico: estructura con morfología polipoidea o en montículo, con un rango de color desde azulado hasta rojizo que característicamente es fácilmente deformable con la compresión ${ }^{4}$; la coloración no fue de ayuda en el diagnóstico de este caso pero sí presentaba una morfología polipoidea. Debido a que endoscópicamente pueden presentar características dispares en cuanto a tamaño, forma y color, requieren la realización de una prueba de imagen para valorar su extensión, generalmente un tomografía computarizada abdominal o una resonancia magnética abdominal ${ }^{5}$.

El diagnóstico definitivo es histológico, existiendo cuatro tipos principales: I (flebectasia múltiples), II (cavernomatoso), III (capilar simple) y IV (angiomatoso) ${ }^{2,6}$; el caso presentado se correspondía con un hemangioma tipo III, también denominado granuloma piogénico, que suele ser solitario y produce una clínica en forma de anemia debido a pérdidas pequeñas y crónicas, como en el caso expuesto, a diferencia del tipo II o cavernomatoso, que suele producir sangrados agudos manifestados como melenas o hematemesis. Histológicamente el tipo III está bien delimitado, con un vaso nutricio central y extensiones lobulillares irradiadas, secundarias a la proliferación de capilares $^{2,3}$, mientras que el tipo II presenta numerosos espacios vasculares repletos de sangre de forma irregular y de un diámetro anormalmente alto, localizados en la mucosa o submucosa pudiendo en ocasiones extenderse a la superficie serosa a través de la capa muscular ${ }^{2,4}$. El más frecuente es el tipo el tipo II ( $55 \%$ de los casos en algunos estudios $^{3}$ ), siendo nuestro caso un hallazgo si cabe más infrecuente.

Tradicionalmente su tratamiento ha sido quirúrgico mediante duodenectomía, si bien al tratarse de tumores benignos la tendencia actual son los tratamientos mínimamente invasivos como los endoscópicos, preferiblemente resección endoscópica (RME), polipectomía convencional en algunos casos seleccionados, inyección local de etanol, esclerosis endoscópica o embolización ${ }^{7}$. Este caso se trató mediante polipectomía convencional porque las características endoscópicas no hicieron sospechar el diagnóstico. Sin embargo, no fue necesario ningún tratamiento adicional al encontrarse los márgenes sin enfermedad.

Es preciso conocer que estas lesiones pueden ser múltiples y ser la manifestación de enfermedades como la hemangiomatosis diseminada neonatal o los síndromes de Maffucci, de Klippel-Trenaunay y de nevu azul en tetina de goma, que sería necesario descartar ${ }^{6,8}$.

En conclusión, el hemangioma capilar es una lesión vascular, benigna y muy infrecuente a nivel del tubo digestivo. Sin embargo, su conocimiento es relevante dado que puede ser causante de anemia ferropénica, siendo esta una de las indicaciones principales de estudios endoscópicos y su resección el tratamiento definitivo.

\section{BIBLIOGRAFÍA}

1. Hu PF, Chen H, Wang XH, Wang WJ, Su N, SHI B. Small intestinal hemangioma: endoscopic or surgical intervention? A case report and review of literature. World J Gastrointest Oncol 2018; 10: 516-521. https://doi.org/10.4251/ wjgo.v10.i12.516

2. Nishiyama N, Mori H, Kobara H, Fujihara S, NoMURA T, KoBAYASHI M et al. Bleeding duodenal hemangioma: morphological changes and 
endoscopic mucosal resection. World J Gastroenterol 2012; 18: 2872-2876. https://doi. org/10.3748/wjg.v18.i22.2872

3. Calvo AM, Erce R, Montón S, Martínez A, Otero A. Hemangioma cavernoso de intestino delgado: una causa rara de obstrucción intestinal. An Sist Sanit Navar 2003; 26: 437-440. https://doi.org/10.4321/s113766272003000500011

4. Takase N, Fukui K, Tani T, Nishimura T, Tanaka T, HARAdA N et al. Preoperative detection and localization of small bowel hemangioma: two case reports. World J Gastroenterol 2017; 23 : 3752-3757. https://doi.org/10.3748/wjg.v23. i 20.3752

5. Durer C, Durer S, Sharbatji M, Comba IY, AhaRONI I, MAJEed U. Cavernous hemangioma of the small bowel: a case report and literature review. Cureus 2018; 10: e3113. https://doi. org/10.7759/cureus.3113

6. Gómez-Galán S, Mosquera-Paz MS, Ceballos J, Cifuentes-Grillo PA, Gutiérrez-Soriano L. Duodenal hemangiolymphangioma presenting as chronic anemia: a case report. BMC Res Notes 2016; 9: 426. https://doi.org/10.1186/ s13104-016-2214-0

7. Kanaji S, NaKamura T, Nishi M, Yamamoto M, Kanemitu K, YamashitTa $\mathrm{K}$ et al. Laparoscopic partial resection for hemangioma in the third portion of the duodenum. World J Gastroenterol 2014; 20: 12341-12345. https://doi. org/10.3748/wjg.v20.i34.12341

8. Coleman J, Phillips R, Steiner R. Small bowel hemangioma in a 2-year-old female with recurrent anemia. Ochsner J 2018; 18: 428-432. https://doi.org/10.31486/toj.18.0099 